\title{
A comparison of selected dosimetric parameters of two Hodgkin Lymphoma radiotherapy techniques with reference to potential risk of radiation induced heart disease
}

\author{
Elias AliBEYKI ${ }^{1}$, Saeid KARIMKHANI ${ }^{2}$, Sepide SAAdATMAND ${ }^{3}$, Parvaneh SHOKRANI ${ }^{1 \text {, a }}$ \\ ${ }^{I}$ Medical Physics Dept, School of Medicine, Isfahan University of Medical Sciences, Isfahan, Iran \\ ${ }^{2}$ Department of Radiation Oncology, Sadra RT Center, Qom, Iran \\ ${ }^{a}$ E-mail address: shokrani@med.mui.ac.ir, shokrani14@yahoo.com
}

(received 2 March 2018; revised 19 June and 27 June 2018; accepted 28 June 2018)

\begin{abstract}
Purpose: Hodgkin lymphoma (HL) is one of the most frequent malignancies among pediatric patients. One of the common causes of death in HL survivors after radiation therapy (RT), is radiation-induced heart disease (RIHD). The aim of this study was to compare several dosimetric parameters for two methods of early stage Hodgkin lymphoma radiotherapy with reference to potential risk of RIHD.

Materials and Methods: Using a series of computed tomography slices of 40 young patients, treatment planning was done in two methods of HL RT, including involved field (IFRT) and involved site (ISRT) in doses of 20, 30, and 35 Gy. Contouring of clinical target volume as well as the organs at risk, including the heart, was performed by a radiation oncologist. The mean and maximum dose of heart $\left(D_{\text {heart-mean }}\right.$ and $\left.D_{\text {heart-max }}\right)$, the volume of heart receiving a dose more than $25 \mathrm{~Gy}$ (V25), and the standard deviation of dose as a dose homogeneity index in heart, were used to compare the RIHD risk.

Results: The mean value for $\mathrm{D}_{\text {heart-mean }}$ in ISRT method in all doses was less compare to IFRT. Maximum reduction in mean value of $D_{\text {heart-mean }}$ occurred at moving from 30 Gy IFRT to ISRT by $9.53 \mathrm{~Gy}$ ( $\mathrm{p}<0.001$ ) and minimum was between 35 Gy IFRT and ISRT. The mean value for $D_{\text {heart-max }}$ was fewer in IFRT rather than ISRT and the maximum difference was between 35 Gy IFRT and ISRT (1.35 Gy). The mean of V25 of heart was $26.66 \%$ and $23.74 \%$ in 35 Gy IFRT and ISRT, respectively, and dose distribution was more homogeneous in IFRT.

Conclusions: If $\mathrm{D}_{\text {heart-max }}$ and $\mathrm{V} 25$ of heart or homogeneity of dose distribution in heart are considered as determining factors in RIHD, then IFRT can be considered optimum, especially in 35 Gy IFRT; while, assuming the $D_{\text {heart-mean }}$ as the most important factor in RIHD, superiority of ISRT over IFRT is observed.
\end{abstract}

Key words: cardiac toxicity; Hodgkin lymphoma; treatment planning; radiotherapy.

\section{Introduction}

Hodgkin lymphoma (HL) is the third most common malignancy among children. Radiotherapy alone or in combination with chemotherapy is widely used in the management of $\mathrm{HL}$, and it is the most effective treatment modality for local controlling of these patients [1]. About 80 percent of patients survive Hodgkin lymphoma event-free, and the 10-year overall survival rate is predicted to fall between $85 \%$ to $97 \%$, depending on sex, for early-stage and between $70 \%$ to $90 \%$ for advanced-stage pediatric HL [2]. Despite the existing efficient treatment regimes, there are some concerns about radiotherapy-induced acute and chronic complications which may reduce patients' quality of life and even survival after the treatment [3]. Cardiovascular disease is known as one of the mortality causes induced by radiotherapy in HL survivors $[1,4,5]$. Different dosimetric parameters have been reported as indexes of cardiac toxicity risk, such as maximum and mean dose of heart, inhomogeneity in dose distribution of heart and the volume of heart that receives high doses [6-11]. There has been extensive research regarding relative risk (RR) of radiation induced heart-related deaths among HL patients of different age ranges. In the age range of 15-20 years, RR range is reported as 2.8-6.8 [4,12-14]. Hence, to improve the overall treatment, RT is mostly used in combination with chemotherapy $[1,3]$. Moreover, different treatment methods in RT are used in order to reduce the irradiated volume and to reduce the dose delivered to the heart and the risk of damage to the heart can be decreased [15]. The clinical target volume (CTV) is defined differently in different HL radiotherapy methods. These methods include extended field radiotherapy (EFRT), involved field radiotherapy (IFRT), involved site radiotherapy (ISRT), and involved node radiotherapy (INRT). Except of EFRT, the other methods are used after chemotherapy [1]. 
In IFRT, the recommended method in combined treatment modality from German Hodgkin Study Group (GHSG) [16], CTV generally includes the regions initially bearing disease defined through the prechemotherapy computerized tomography (CT) or positron emission tomography (PET) images [17]. In INRT, the CTV encompasses only the involved nodes plus a margin and Gross tumor volume (GTV) is outlined using images of prechemotherapy high quality CT and PET/CT and planning is performed by fused images of pre and postchemotherapy. However, when fusing pre and post chemotherapy images is not possible, the clinician in ISRT can specify CTV by adding a larger margin in the prechemotherapy images to include all uncertainties in GTV definition $[3,18]$. Implementation of INRT and ISRT reduces the treatment volume considerably, and there are recommendations to replace INRT and ISRT instead of IFRT. However, according to some studies, the available evidences are insufficient to support or refute the definite advantage of INRT or ISRT over IFRT [19]. It was also demonstrated there is no difference in progression-free survival (PFS) and overall survival (OS) when applying INRT compared to IFRT or EFRT [20].

In a study that IFRT method was used instead of mantle field with the same prescribed dose, 35 and $20 \mathrm{~Gy}$, the mean doses of the whole heart were 17.2 and $9.9 \mathrm{~Gy}$, respectively [21]. In another study, the results indicated the dose of heart is considerably decreased by moving from IFRT to INRT [22]. Using imaging information such as pre and postchemotherapy CT and PET/CT images, a TPS with accurate algorithm, and patient immobilizing devices, different treatment plans can be designed and delivered. Although the lowest mean dose to heart has been reported for INRT [22], the required diagnostic and treatment modalities may not be available in some of the developing countries. In such cases, the treatment options are ISRT and IFRT. In our department, HL patients have access to PET-CT imaging modality but there are limited resources for accurate fusion of PET-CT images for treatment planning purposes. Hence the INRT method is not an available option. The goal of this study was to evaluate and to compare several dosimetric parameters for two methods of early stage Hodgkin lymphoma radiotherapy (patients who had both mediastinum and cervical nodes involvement with or without axillary nodes involvement) with reference to potential risk of radiation induced heart disease.

\section{Materials and Methods}

\section{Patient selection}

In this study 40 young patients (with an average age of 21 years) with mediastinum and cervical nodes involvement (with or without axillary nodes involvement) (stage 2, based on Ann Arbor staging system [23]) were chosen. They were previously treated and their archived CT scan images were used for treatment planning.

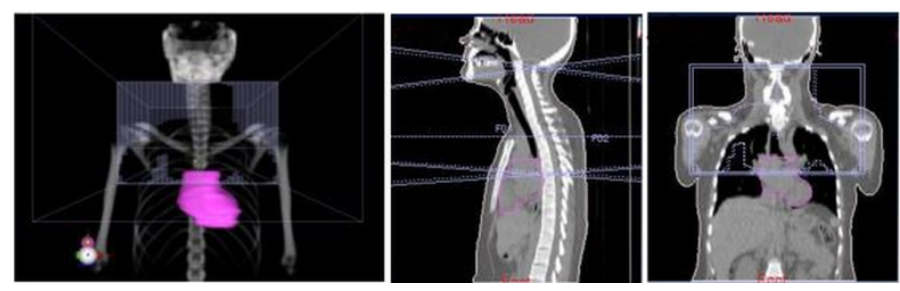

Figure 1. An example of beam's eye view (BEV). Coronal and sagittal plane in ISRT.

\section{Prescribed dose}

Doses of 35, 30, and 20 Gy were prescribed at the isocenter in each methods. The isocenter point and dose weight of each fields were specified based on obtaining a uniform dose coverage in the PTV ( $\geq 95 \%$ of the prescribed dose) [24].

\section{Contouring and treatment planning}

An ISOgray, version: 4.3.1.23L commercial treatment planning system (TPS) which had been commissioned for 6 and $18 \mathrm{MV}$ photons generated by an Elekta Synergy Platform linear accelerator was used for contour delineations, treatment planning and dose calculations. The TPS uses a collapsed cone convolution algorithm for computing the dose.

As discussed earlier, the CTV is defined differently in different HL radiotherapy methods. These methods include EFRT, IFRT, ISRT, and INRT. In IFRT, CTV generally includes the regions initially bearing disease defined through the prechemotherapy images (CT or PET) [17]. In INRT, the CTV encompasses only the involved nodes plus a margin and Gross tumor volume (GTV) is outlined using images of prechemotherapy high quality CT and PET/CT and planning is performed by fused images of pre and postchemotherapy. However, when fusing pre and post chemotherapy images is not possible, the clinician in ISRT can specify CTV by adding a larger margin in the prechemotherapy images to include all uncertainties in GTV definition [3,18].

According to available facilities, two methods of IFRT and ISRT were applied in the study. The contouring of CTV and OAR's including heart was done by an experienced radiation oncologist. CTV for ISRT (CTVIS) encompassed the initially involved sites, detected based on the prechemotherapy imaging (CT and PET/CT); Then, it was expanded cranio-caudally by $1.5 \mathrm{~cm}$ in the lymphatic spread direction which resulted in a coplanar AP/PA field as shown in Figure 1. In the transverse plane, it was not necessary to include the entire nodal regions and $1 \mathrm{~cm}$ margin was enough [18].

For patients in this study, CTV delineation for IFRT (CTVIF) was done in several phases. First, CTV included all cervical, infra and supraclavicular, mediastinal, axillary, and hilar lymph nodes treated by using two coplanar AP/PA mantle fields. Hence, the whole heart, except for a part of heart apex (shielded based on the physician discretion) was placed in the mantle field (Figure 2a). In this step, 14.4 Gy was prescribed to CTV. Afterwards, uninvolved paracardiac nodes were 
shielded and treatment dose was increased to 20, 30 and 30.6 Gy for studies with prescribed doses of 20, 30 and $35 \mathrm{~Gy}$ (Figure 2b). For studies using dose of $35 \mathrm{~Gy}$, after delivering of 30.6 Gy to CTV, the whole heart was blocked (Figure 2c) and the rest of the AP/PA mantle fields was treated up to $35 \mathrm{~Gy}$ [17]. In each of the methods, PTV was constructed from CTV by adding $1 \mathrm{~cm}$ margin in the cervical region, and in the mediastinum, $1.5 \mathrm{~cm}$ margin in the cranio-caudal direction and $1 \mathrm{~cm}$ in the transverse direction [18,25]. In all methods, $6 \mathrm{MV}$ and $18 \mathrm{MV}$ photon beams were chosen for AP and PA fields, respectively. ISRT shaping and shielding were done by an 80leaf multileaf collimator (MLC) and because of limitations on island blocking by MLC in the mantle field, IFRT shielding was done by custom blocking.

(a)

(b)
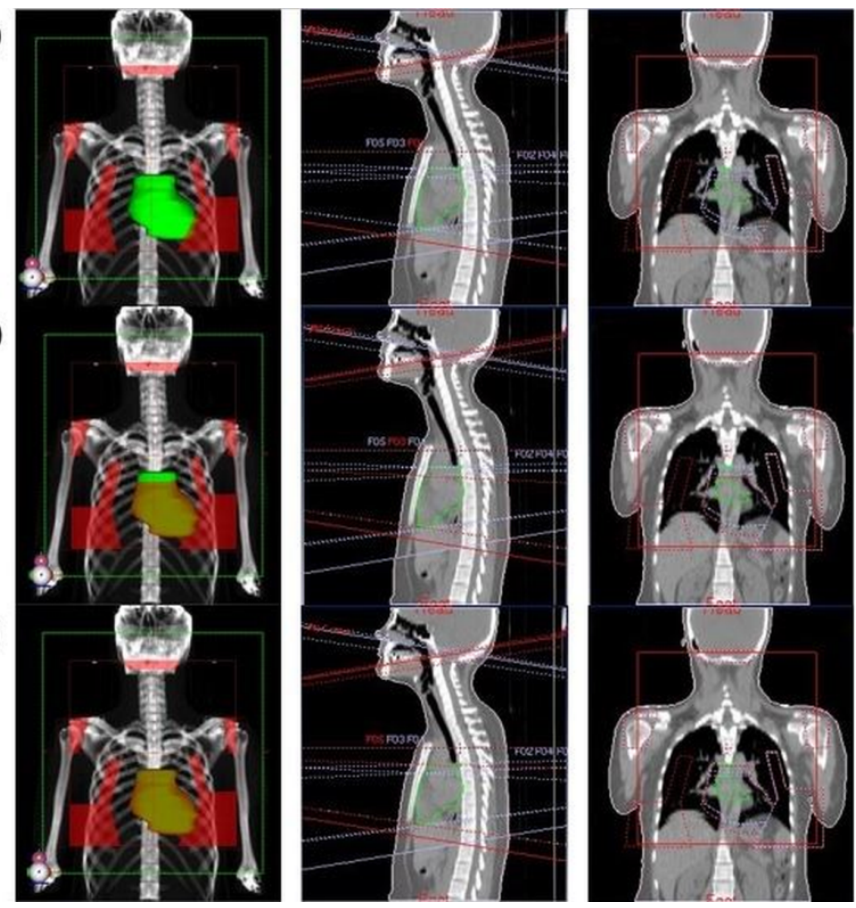

Figure 2. An example of Beam's eye view (BEV). Coronal and sagittal plane, in IFRT. Mantle field in phase 1 (a), sparing uninvolved paracardiac lymph nodes in phase 2 (b), and whole heart blocking in phase 3 (for studies using dose of $35 \mathrm{~Gy}$ ) of the treatment (c).

\section{Dosimetric parameters}

Mean dose $\left(D_{\text {heart-mean }}\right)$, maximum dose $\left(D_{\text {heart-max }}\right)$, V25 of heart (Vx: Volume of the organ receiving $\geq \mathrm{x}$ Gy) and standard deviation (Std. Deviation) of dose distribution (as an inhomogeneity index) were chosen as dosimetric parameters that can be used to express risk of radiation induced-cardiac disease $[6,8,10,11]$ in IFRT and ISRT method.

\section{Statistical test}

In this study, SPSS software package was used for statistical analysis. For datasets with normal distribution, the paired sample t-test was used; and for those datasets which could not be assumed to be normally distributed, the Wilcoxon test was used.

\section{Results}

Using the dose volume histogram (DVH) of heart, $\mathrm{D}_{\text {heart-mean, }}$ $\mathrm{D}_{\text {heart-max }}, \mathrm{V} 25$, and Std. deviation of heart dose distribution in each treatment plan were estimated (Table 1).

Except for $\mathrm{D}_{\text {heart-max }}$ in IFRT and ISRT using total dose of 20 Gy (IFRT-20 and ISRT-20 respectively), and V25 in IFRT and ISRT using 30 Gy (IFRT-30 and ISRT-30 respectively), significant differences were observed when comparing other dosimetric parameters in different methods.

The mean of $\mathrm{D}_{\text {heart-mean }}$ in ISRT method in all prescribed doses (20, 30, and $35 \mathrm{~Gy}$ ) was lower compared to IFRT. Maximum reduction in mean $\mathrm{D}_{\text {heart-mean }}$ occurred at moving from IFRT-30 to ISRT-30 by $9.53 \mathrm{~Gy}$ ( $\mathrm{p}<0.001$ ) and minimum was between 35 Gy IFRT and ISRT (IFRT-35 and ISRT-35 respectively). The mean $D_{\text {heart-max }}$ of heart in IFRT was lower compared to ISRT and the maximum difference was between IFRT-35 and ISRT-35 (1.35 Gy). The mean of V25 of heart was $26.66 \%$ and $23.74 \%$ in ISRT-35 and IFRT-35, respectively. The mean of std. deviation of dose distribution in volume of heart in IFRT was lower compared to ISRT for all prescribed doses (more details in Table 1).
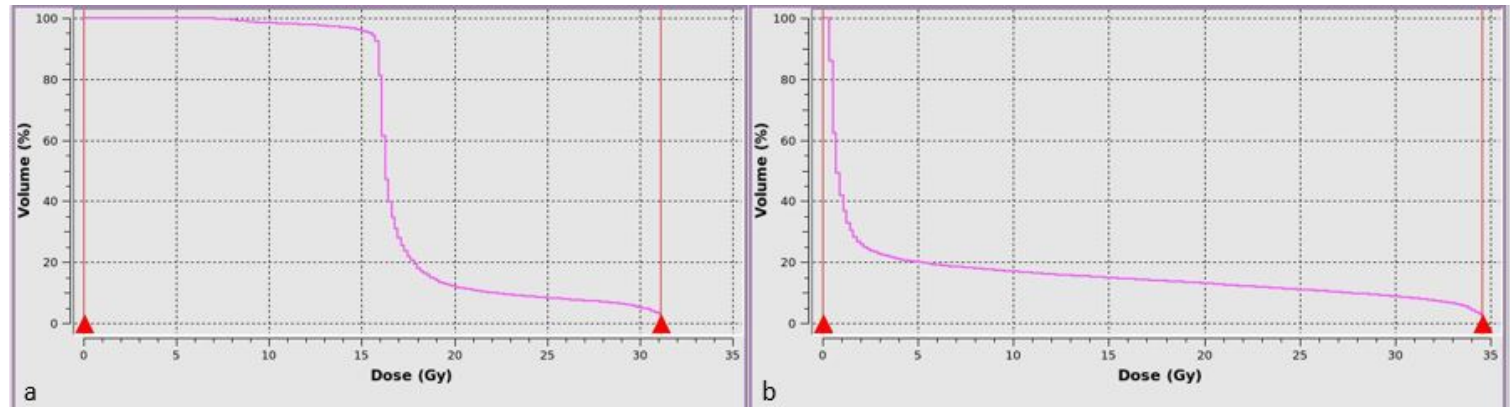

Figure 3. Examples of exported cumulative DVHs for heart in IFRT (a), and ISRT (b) in prescribed dose of 35 Gy. DVH in IFRT is computed as the net of three phases of treatment. 
Table 1. Dosimetric data related to the heart in each method.

\begin{tabular}{|c|c|c|c|c|c|c|}
\hline Dosimetric Parameter & Method & Minimum & Maximum & Mean & SD $^{\mathrm{a}}$ & P-Value \\
\hline \multirow{6}{*}{$D_{\text {heart-max }}(\mathbf{G y})$} & ISRT-20 & 17.95 & 23.27 & 21.59 & 1.81 & \multirow{2}{*}{$0.500^{\mathrm{c}}$} \\
\hline & IFRT-20 & 19.43 & 22.08 & 21.78 & 2.19 & \\
\hline & ISRT-30 & 26.78 & 34.67 & 31.52 & 1.30 & \multirow{2}{*}{$0.001^{\mathrm{c}}$} \\
\hline & IFRT-30 & 28.27 & 33.82 & 31.26 & 0.94 & \\
\hline & ISRT-35 & 31.04 & 40.15 & 36.44 & 1.63 & \multirow{2}{*}{$<0.001^{\mathrm{c}}$} \\
\hline & IFRT-35 & 31.66 & 38.72 & 35.09 & 1.66 & \\
\hline \multirow{6}{*}{$\mathbf{D}_{\text {heart-mean }}(\mathbf{G y})$} & ISRT-20 & 0.61 & 14.26 & 6.91 & 3.65 & \multirow{2}{*}{$<0.001^{\circ}$} \\
\hline & IFRT-20 & 14.49 & 18.27 & 16.15 & 1.02 & \\
\hline & ISRT-30 & 0.91 & 21.35 & 9.64 & 5.37 & \multirow{2}{*}{$<0.001^{\mathrm{c}}$} \\
\hline & IFRT-30 & 15.22 & 24.28 & 19.17 & 2.53 & \\
\hline & ISRT-35 & 1.06 & 24.75 & 11.82 & 6.56 & $<O \Omega 01^{\mathrm{c}}$ \\
\hline & IFRT-35 & 15.54 & 25.57 & 19.75 & 2.72 & -0.001 \\
\hline \multirow{4}{*}{ V25 (\%) } & ISRT-30 & 0.11 & 62.32 & 21.64 & 13.63 & \multirow{2}{*}{$0.676^{d}$} \\
\hline & IFRT-30 & 0.51 & 60.90 & 22.42 & 16.29 & \\
\hline & ISRT-35 & 0.25 & 64.56 & 26.66 & 17.52 & $1^{d}$ \\
\hline & IFRT-35 & 1.27 & 61.60 & 23.74 & 16.22 & 0.001 \\
\hline \multirow{6}{*}{ Std. Deviation $^{\text {b }}(\mathbf{G y})$} & ISRT-20 & 1.70 & 11.87 & 7.23 & 1.97 & \multirow{2}{*}{$<0.001^{\mathrm{d}}$} \\
\hline & IFRT-20 & 1.78 & 5.21 & 3.06 & 0.75 & \\
\hline & ISRT-30 & 2.55 & 14.00 & 10.49 & 2.70 & \multirow{2}{*}{$<0.001^{\mathrm{d}}$} \\
\hline & IFRT-30 & 2.60 & 8.73 & 5.84 & 1.53 & \\
\hline & ISRT-35 & 2.96 & 16.29 & 12.19 & 3.13 & \multirow{2}{*}{$<0.001^{\mathrm{d}}$} \\
\hline & IFRT-35 & 2.88 & 9.66 & 6.30 & 1.64 & \\
\hline
\end{tabular}

a: SD represents the Standard deviation of patients' data. $\boldsymbol{b}$ : Std. Deviation of heart dose distribution (as an inhomogeneity index), one of the dosimetric parameters in RIHD. Statistical tests: Paired t test (c) and Wilcoxon test (d)

\section{Discussion}

In this study, using CT images of 40 young Hodgkin lymphoma patients, dose distributions for two methods of radiation treatment planning was calculated. Following dosimetric parameters; $\mathrm{D}_{\text {heart-mean, }} \mathrm{D}_{\text {heart-max }}, \mathrm{V} 25$, and dose inhomogeneity index were calculated and were used for plan evaluations.

Results showed that the mean of $\mathrm{D}_{\text {heart-mean was lowest in }}$ ISRT method against IFRT method. Maximum difference of mean of $\mathrm{D}_{\text {heart-mean }}$ was obtained between IFRT-30 and ISRT-30 (9.53 Gy), and minimum of it occurred in IFRT-35 and ISRT35 (7.93 Gy). Except for $D_{\text {heart-mean, }}$ other dosimetric parameters ( $\mathrm{D}_{\text {heart-max }}, \mathrm{V} 25$, and Std. deviation) indicated the superiority of IFRT to ISRT, especially in IFRT-35. Although this superiority was mild, these dosimetric parameters are considered as dosimetric indexes in RIHD [6,8,10].

In a study, the relationship between radiation doses and the relative risk (RR) of death were analyzed and the best dose response model was reported as a function of $\mathrm{D}_{\text {heart-mean }}(\mathrm{RR}$ of RIHD was 0.6 (95\% confidence interval [CI], 0.2 to 2.5 ) at $1 \mathrm{~Gy}$ of the mean dose delivered to the heart) [11]. Also, the mean radiation dose to the heart has been indicated as an important risk factor of the development of coronary heart disease (CHD) in HL survivors [26,27]. However, the importance of the volume of heart that receives high doses has been discussed as well; so that, in a study on a random sample of $125 \mathrm{HL}$ patients treated with mediastinal RT, the volume effect was known as the major dosimetric parameter in the CHD (Hazard Ratio [HZ] 0.98 and 1.03 for V5 of left anterior descending artery and V20 of left circumflex artery, respectively) [9]. As well, it is predicted that if the volume of heart receiving a dose more than $25 \mathrm{~Gy}$ is less than $10 \%$ of heart $(\mathrm{V} 25<10 \%)$, the probability of cardiac mortality during 15 years after radiotherapy is less than $1 \%$ [7,8]. Moreover, there are studies indicating more inhomogeneity of dose distribution in the heart caused the greater risk of late cardiac effects and asymmetric development [9,10]; so, inhomogeneity of dose distribution is an another important parameter in RIHD, especially in young patients. By available evidences, it is difficult to judge what method is optimum (IFRT vs ISRT), because the author(s) did not find any comprehensive model to explain contribution of each parameter to the induction of RIHD. The results of this research are limited to 40 patients with mediastinum and cervical nodes involvement. In these patients, IFRT planning is done in several treatment phases, (IFRT-30 and IFRT-20 in two phases, and IFRT-35 in three phases) and CTV delineation and field design is done differently in each phase. A cohort study is required in order to assess the relationship between the dosimetric factors and the risk of RIHD, and to select one factor among the mean dose, V25, or max dose as the most influential factor for predicting the probability of RIHD.

\section{Conclusions}

Depending on the extent of disease and availability of patient clinical information, such as pre and postchemotherapy CT and PET/CT images, different radiation treatment plans can be designed and delivered for HL patients. Although the lowest 
mean dose to heart has been reported in INRT and ISRT; the required diagnostic and treatment modalities may not be available in developing countries. This study showed that the comparison of two methods for radiotherapy of Hodgkin Lymphoma, i.e. ISRT and IFRT, produce different values of dosimetric parameters. Assuming the mean dose of heart as the most important factor in RIHD, potential risk of RIHD is less in ISRT. While, if $\mathrm{D}_{\text {heart-max }}$ and V25 of heart or homogeneity of dose distribution in heart are considered as determining factors for risk of RIHD, then IFRT can be considered optimum for the studied patients especially when 35 Gy is prescribed.

\section{Acknowledgements}

The authors are thankful to Dr. Fateme Zahedi and Ali Khodadadi for helping in progress of work. This work was performed at Sadra Radiation Therapy Center, Farjad Group, Qom, Iran, as a part of master's dissertation (Elias Alibeyki), MSc student of medical physics, Department of Medical Physics \& Medical Engineering, Isfahan University of Medical Sciences, Isfahan, Iran.

\section{Source of Support}

Isfahan University of Medical Sciences

\section{References}

[1] Halperin EC, Brady LW, Perez CA, et al. Perez \& Brady's Principles and Practice of Radiation Oncology. Wolters Kluwer Health; 2013.

[2] Donaldson SS, Hudson MM, Lamborn KR, et al. VAMP and low-dose, involved-field radiation for children and adolescents with favorable, early-stage Hodgkin's disease: results of a prospective clinical trial. J Clin Oncol. 2002;20(14):3081-3087.

[3] Specht L, Yahalom J, Illidge T, et al. Modern radiation therapy for Hodgkin lymphoma: field and dose guidelines from the international lymphoma radiation oncology group (ILROG). Int J Radiat Oncol Biol Phys. 2014;89(4):854-862.

[4] Aleman BM, van den Belt-Dusebout AW, Klokman WJ, et al. Long-term cause-specific mortality of patients treated for Hodgkin's disease. J Clin Oncol. 2003;21(18):3431-3439.

[5] $\mathrm{Ng}$ AK. Review of the cardiac long-term effects of therapy for Hodgkin lymphoma. Br J Haematol. 2011;154(1):23-31.

[6] Baker JE, Moulder JE, Hopewell JW. Radiation as a risk factor for cardiovascular disease. Antioxid Redox Signalg. 2011;15(7):1945-1956.

[7] Emami B. Tolerance of normal tissue to therapeutic radiation. Rep Rradiother Oncol. 2013;1(1):123-127.

[8] Gagliardi G, Constine LS, Moiseenko V, et al. Radiation dose-volume effects in the heart. Int J Radiat Oncol Biol Phys. 2010;76(3):S77-S85.

[9] Hahn E, Jiang H, Ng A, et al. Late Cardiac Toxicity After Mediastinal Radiation Therapy for Hodgkin Lymphoma: Contributions of Coronary Artery and Whole Heart Dose-Volume Variables to Risk Prediction. Int J Radiat Oncol Biol Phys. 2017;98(5):1116-1123.

[10] Jakacki RI, Goldwein JW, Larsen RL, et al. Cardiac dysfunction following spinal irradiation during childhood. J Clin Oncol. 1993;11(6):1033-1038.

[11] Tukenova M, Guibout C, Oberlin O, et al. Role of cancer treatment in long-term overall and cardiovascular mortality after childhood cancer. J Clin Oncol. 2010;28(8):1308-1315.

[12] Hoppe R. Hodgkin's disease: complications of therapy and excess mortality. Ann Oncol. 1997;8(suppl 1):S115-S118.

[13] $\mathrm{Ng} \mathrm{AK}$, Bernardo MP, Weller E, et al. Long-term survival and competing causes of death in patients with early-stage Hodgkin's disease treated at age 50 or younger. J Clin Oncol. 2002;20(8):2101-2108.

[14] Swerdlow AJ, Higgins CD, Smith P, et al. Myocardial infarction mortality risk after treatment for Hodgkin disease: a collaborative British cohort study. J Natl Cancer Inst. 2007;99(3):206-214.

[15] Paumier A, Ghalibafian M, Beaudre A, et al. Involved-node radiotherapy and modern radiation treatment techniques in patients with Hodgkin lymphoma. Int J Radiat Oncol Biol Phys. 2011;80(1):199-205.

[16] Engert A, Pluetschow A, Eich HT, et al. Combined Modality Treatment of Two or Four Cycles of ABVD Followed by Involved Field Radiotherapy in the Treatment of Patients with Early Stage Hodgkin's Lymphoma: Update Interim Analysis of the Randomised HD10 Study of the German Hodgkin Study Group (GHSG). Blood. 2005;106(11):2673.

[17] Yahalom J, Mauch P. The involved field is back: issues in delineating the radiation field in Hodgkin's disease. Ann Oncol. 2002;13(suppl 1):79-83.

[18] Hoskin P, Díez P, Williams M, et al. Recommendations for the use of radiotherapy in nodal lymphoma. Clin Oncol. 2013;25(1):4958.

[19] Herst J, Crump M, Baldassarre F, et al. Management of Early-stage Hodgkin Lymphoma: A Practice Guideline. Clin Oncol. 2017;29(1):e5-e12.

[20] Campbell BA, Voss N, Pickles T, et al. Involved-nodal radiation therapy as a component of combination therapy for limited-stage Hodgkin's lymphoma: a question of field size. J Clin Oncol. 2008;26(32):5170-5174. 
[21] Koh E-S, Tran TH, Heydarian M, et al. A comparison of mantle versus involved-field radiotherapy for Hodgkin's lymphoma: reduction in normal tissue dose and second cancer risk. Radiat Oncol. 2007;2:13.

[22] Koeck J, Abo-Madyan Y, Lohr F, et al. Radiotherapy for early mediastinal Hodgkin lymphoma according to the German Hodgkin Study Group (GHSG): the roles of intensity-modulated radiotherapy and involved-node radiotherapy. Int J Radiat Oncol Biol Phys. 2012;83(1):268-276.

[23] Sobin LH, Gospodarowicz MK, Wittekind C. TNM Classification of Malignant Tumours. Wiley; 2011.

[24] Khan FM, Gibbons JP. Khan's The Physics of Radiation Therapy. Wolters Kluwer Health; 2014.

[25] Barrett A, Dobbs J, Roques T. Practical Radiotherapy Planning Fourth Edition. Taylor \& Francis; 2009.

[26] Carr ZA, Land CE, Kleinerman RA, et al. Coronary heart disease after radiotherapy for peptic ulcer disease. Int J Radiat Oncol Biol Phys. 2005;61(3):842-850.

[27] van Nimwegen FA, Schaapveld M, Cutter DJ, et al. Radiation dose-response relationship for risk of coronary heart disease in survivors of Hodgkin lymphoma. J Clin Oncol. 2015;34(3):235-243. 